\title{
Biologia reprodutiva de Dipsas neivai Amaral e D. catesbyi (Sentzen) (Serpentes, Colubridae) no sudeste da Bahia, Brasil
}

\author{
Fátima Q. Alves ${ }^{1}$, Antônio J.S. Argolo² \& Jorge Jim ${ }^{3}$ \\ ${ }^{1}$ Rua D, Loteamento Santa Teresa 34, Banco Raso, 45600-000 Itabuna, Bahia, Brasil. E-mail: fqueiroz48@hotmail.com \\ 2 Departamento de Ciências Biológicas, Universidade Estadual de Santa Cruz. Rodovia Ilhéus-Itabuna Km 16, 45650-000 \\ Ilhéus, Bahia, Brasil. E-mail: lachesis@uesc.br \\ ${ }^{3}$ Departamento de Zoologia Universidade Estadual Paulista. Rubião Júnior, 18618-000 Botucatu, São Paulo, Brasil.
}

\begin{abstract}
Reproductive biology in Dipsas neivai and D. catesbyi (Serpentes, Colubridae) in southeastern Bahia, Brasil. The reproductive biology of Dipsas neivai Amaral, 1923 and Dipsas catesbyi (Sentzen, 1796), was studied by dissecting 261 specimens of $D$. neivai and 222 of $D$. catesbyi. In D. neivai males mature at smaller body size than females, and in D. catesbyi the opposite was observed. The females were larger than males in both species. The clutch size ranged from one to eight in D. neivai, and one to six in D. catesbyi, but it is not correlated with female length in the last one. Reproductive cycles in both males and females are aseasonal, with vitelogensis and spermatogenesis occurring throughout the year. The couple in females of $D$. neivai is dissociated from the vitellogenesis. D. neivai and D. catesbyi are syntopic and reproduce in the same time of the year, which is possibly related to the continuous availability of prey and little climate variation in southern Bahia.

KEY WORDS. Atlantic forest, gonadal cycle, sexual dimorphism, sexual maturity.
\end{abstract}

RESUMO. A biologia reprodutiva de Dipsas neivai Amaral, 1923 e Dipsas catesbyi (Sentezen, 1796) foi estudada através da dissecção de 261 espécimes de D. neivai e 222 de D. catesbyi. Em D. neivai o macho amadurece com menor tamanho do que a fêmea, ocorrendo o oposto em $D$. catesbyi. Em ambas as espécies a fêmea atinge maior tamanho corporal que o macho. O tamanho da ninhada variou de um a oito ovos em $D$. neivai e de um a seis em D. catesbyi, não estando correlacionado com o tamanho da fêmea na última. $O$ ciclo reprodutivo em ambos machos e fêmeas é contínuo, com vitelogênese e espermatogênese ocorrendo ao longo do ano todo. Em Dipsas neivai, a cópula nas fêmeas é dissociado da vitelogênese. $D$. neivai e $D$. catesbyi são sintópicas, e reproduzem no mesmo período do ano, o que possivelmente está relacionado à disponibilidade contínua de presas e à pouca variação climática no sudeste da Bahia.

PALAVRAS CHAVE. Ciclo gonadal, dimorfismo sexual, Floresta Atlântica, maturidade sexual.

Serpentes Dipsas Laurenti, 1768, constituem um gênero bastante diversificado de colubrídeos de florestas, distribuídas desde o México até o sul do Brasil (Peters \& Orejas-Miranda 1970). São denominadas popularmente "dormideira", "dorminhoca" ou "come-lesma" (Amaral 1926, 1978), além de "cobra-cipó", na Amazônia (Cunha \& Nascimento 1978). Apesar de inofensivas são bastante temidas no sudeste da Bahia, onde são conhecidas como "pingo-de-ouro" (Dipsas indica indica Laurenti, 1768, Dipsas indica petersi Hoge \& Romano, 1975) ou "jaracuçu" (D. neivai) (ARGÔLo 2004). Embora ocupem razoável extensão geográfica, são serpentes pouco representadas em coleções (CuNHA \& Nascimento 1978, Duellman 1978, Porto \& Fernandes 1996) e dados reprodutivos consistentes são disponíveis para poucas espécies (Hartmann et al. 2002). Dipsas albifrons (Sauvage, 1884) constitui uma das poucas formas do gênero razoavelmente bem representadas em coleções, mas sua distribuição abrange principalmente áreas de altas latitudes na Mata Atlântica. No momento, é a única Dipsas do bioma que tem o ciclo reprodutivo caracterizado (HARTMANn et al. 2002). Nenhuma congênere de áreas mais setentrionais da Mata Atlântica foi estudada com base em número significativo de espécimes.

O sudeste da Bahia abriga cinco espécies de Dipsas, das quais $D$. neivai e $D$. catesbyi são relativamente freqüentes em cacauais (ARgôlo 2004). D. neivai distribui-se de Alagoas à Santa Catarina, mas, apesar da distribuição ampla, é pouco representada nas principais coleções herpetológicas do país (PORTo \& Fernandes 1996). Estes autores sugeriram reprodução contínua em $D$. neivai, com base em indivíduos de diferentes latitudes da Mata Atlântica (Porto \& Fernandes 1996). D. catesbyi apresenta ampla distribuição na Amazônia e uma população isola- 
da na Mata Atlântica do sudeste da Bahia (Argôlo 2004). Considera-se que as populações de Iquitos, no Peru, possuem reprodução contínua, mas não existem dados suficientes de outras áreas amazônicas (Zug et al. 1979) e nada se conhece sobre as populações do sudeste da Bahia.

Dentre os colubrideos, os Dipsadini são serpentes de tamanho médio ou pequeno, cujas fêmeas são maiores que os machos (Fiтch 1981). A despeito da grande diversidade ecológica na família (Fitch 1981), diversos estudos (e.g. Parker \& Plummer 1987, Shine 1993, Pizzatto \& Marques 2002, Hartmann et al. 2002, Jordão \& Bizerra 1996, Marques \& PUORTo 1998) identificaram o mesmo padrão de dimorfismo de outros grupos.

O dimorfismo sexual de tamanho está relacionado com a latitude (SHINE 1994) e a distribuição geográfica é marcante na determinação não apenas desta, mas de outras características reprodutivas (SHINe 1994, HarTmann et al. 2002). Assim, conclusões consistentes acerca de tais tópicos para espécies amplamente distribuídas devem apoiar-se em dados ao longo da sua distribuição. D. neivai e $D$. catesbyi constituem bons modelos para uma abordagem dessa natureza. A primeira, por ocorrer em diferentes latitudes da Mata Atlântica e a segunda por apresentar considerável disjunção entre este bioma e a Amazônia. Neste trabalho apresentamos dados de maturidade, dimorfismo sexual de tamanho e ciclo reprodutivo de D. neivai e D. catesbyi no sudeste da Bahia.

\section{MATERIAL E MÉTODOS}

\section{Área de estudo}

Os espécimes foram coletados entre 1986 e 1998 principalmente na faixa de floresta ombrófila densa do sudeste da Bahia, onde predomina o cultivo do cacau (Theobroma cacao). A área situa-se entre a costa Atlântica e aproximadamente a $41^{\circ} \mathrm{W}$, e $13^{\circ} 00^{\prime}-18^{\circ} 15^{\prime} \mathrm{S}$ (Fig. 1). Nessa faixa, o relevo em grande parte é suavemente ondulado e o clima é do tipo Af (Koeppen), quente e úmido, sem estação seca definida (Roeder 1975). O índice pluviométrico médio anual atinge $1.500 \mathrm{~mm}$. A temperatura média mensal oscila entre 20 e $26^{\circ} \mathrm{C}$ e a anual em torno de $24^{\circ} \mathrm{C}$. No verão a temperatura média é $26^{\circ} \mathrm{C}$ e no inverno $18^{\circ} \mathrm{C}$. Os meses mais quentes são outubro a abril e os mais frios, junho a agosto. A umidade situa-se em torno de $78 \%$ atingindo índices médios superiores a $80 \%$ próximo ao litoral. Os níveis elevados de umidade e temperatura, associados à ocorrência quase constante de condensação, ocasionam precipitações relativamente uniformes e abundantes ao longo do ano, com valores que superam os $2.000 \mathrm{~mm}$ em algumas localidades do litoral (SÁ et al. 1982). Apenas seis indivíduos de D. neivai foram obtidos em área de floresta estacional semidecidual, onde a precipitação média anual situa-se em torno de $1.000 \mathrm{~mm}$ e ocorre um período seco (tipo climático Am na classificação de Koeppen).

Foram analisados 261 indivíduos de $D$. neivai e 222 de $D$. catesbyi da Coleção Zoológica Gregório Bondar (CZGB) da Comissão Executiva do Plano da Lavoura Cacaueira (CEPEC/ CEPLAC), em Ilhéus-BA. Os seguintes dados foram obtidos dos

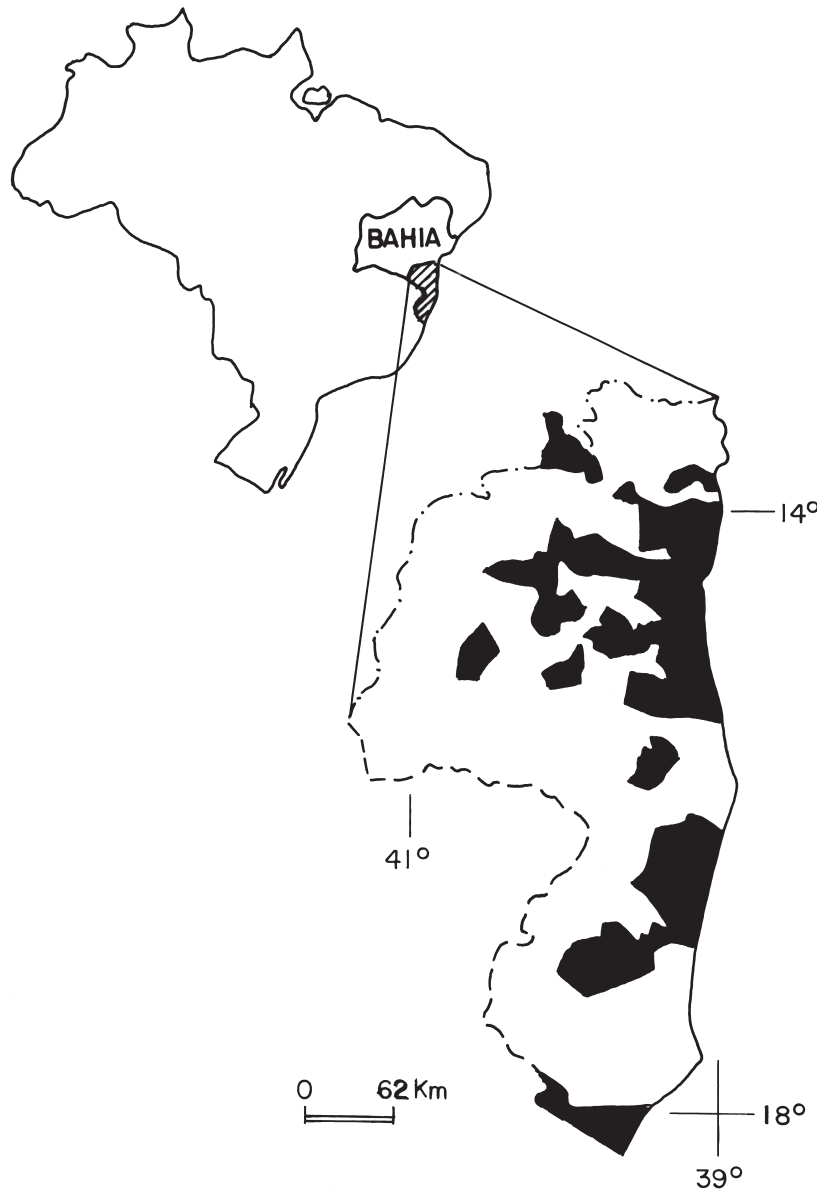

Figura 1. Localização da área de estudo e coletas no sudeste da Bahia.

espécimes dissecados: (1) comprimento rostro-cloacal (CRC); (2) comprimento caudal (CC); (3) diâmetro do maior folículo ovariano; (4) número de folículos e ovos; (5) espessura dos ovários e ovidutos; (6) comprimento do maior ovo (eixo maior); (7) diâmetro dos ductos deferentes (próximo ao epidídimo); (8) comprimento dos testículos (eixo maior; CTE); (7) estágio de enovelamento dos ductos deferentes; (8) maturidade reprodutiva, se estavam maduros ou imaturos. Foram considerados indivíduos maduros as fêmeas com folículos ovarianos a partir da vitelogênese inicial (3-4 mm), ovidutos espessos ou com ovos nos ovidutos; e os machos com testículos desenvolvidos e túrgidos ou ductos deferentes opacos e enovelados, indicando a presença de esperma (SHINe 1977a, b, 1984, Zug et al. 1979).

A variação do ciclo gonadal das fêmeas foi analisada com base no número de fêmeas maduras coletadas em cada mês e o número de fêmeas com folículos vitelogênicos ou ovos no mesmo período, através do teste Qui-quadrado. O comprimento dos testículos e o diâmetro dos ductos deferentes foram correla- 
cionados com o CRC e os resultados utilizados para indicar a presença de atividade espermatogênica (SHINe 1977b). A atividade espermatogênica também foi avaliada pelo exame histológico dos testículos, epidídimos e ductos deferentes. Para análise histológica foram selecionados indivíduos maduros de vários tamanhos e cada mês do ano foi representado por dois ou três espécimes.

O período de desova e eclosão, tamanho da ninhada e dos recém-nascidos foram obtidos a partir de desovas efetuadas em cativeiro (espécimes mantidos no biotério da CEPLAC), dos ovos e filhotes encontrados na natureza e dos espécimes preservados da coleção.

O CRC da fêmea foi correlacionado com o tamanho da ninhada através da análise de correlação. O grau de dimorfismo sexual de tamanho (DST) foi calculado pela seguinte fórmula: a média do CRC do maior sexo dividido pela média do CRC do menor sexo e subtraído de 1 (SHINe 1994). O grau de dimorfismo sexual foi calculado nos jovens para avaliar se este caracter ocorre em toda história de vida ou se há mudança ontogenética.

\section{RESULTADOS}

\section{Maturidade e dimorfismo sexual de tamanho (DST)}

A menor fêmea madura de $D$. neivai mediu $432 \mathrm{~mm}$ de CRC e o menor macho $298 \mathrm{~mm}$. As fêmeas atingiram a maturidade sexual com $52,43 \%$ do CRC e os machos com $42,45 \%$. As fêmeas maduras $(584,41 \pm 86,67 \mathrm{~mm}$; amplitude de variação = $432-820 \mathrm{~mm} ; \mathrm{n}=91$ ) foram significativamente maiores do que os machos maduros $(521,79 \pm 81,29 \mathrm{~mm}$; amplitude de variação = 298-702 mm; $\mathrm{n}=99)(\mathrm{t}=5,12 ; \mathrm{df}=183,83 ; \mathrm{p}<0,05)$. O grau de DST foi maior nos adultos $(0,05)$ que nos jovens $(0,01)$.

Em D. catesbyi, a menor fêmea madura mediu $331 \mathrm{~mm}$ e o menor macho maduro $341 \mathrm{~mm}$. As fêmeas alcançam a maturidade com 51,72\% do CRC e os machos com 59,30\%. As fêmeas maduras $(494,19 \pm 63,91 \mathrm{~mm}$; amplitude de variação = 331$640 \mathrm{~mm} ; \mathrm{n}=54$ ) também foram significativamente maiores do que machos $(463,69 \pm 53,78 \mathrm{~mm}$; amplitude de variação $=341$ $575 ; \mathrm{n}=65)(\mathrm{t}=2,782 ; \mathrm{df}=103,10 ; \mathrm{p}<0,05)$. O grau de DST foi 0,06 nos adultos e nos jovens foi negativo $(-0,06)$.

\section{Ciclo reprodutivo}

\section{Fêmeas}

Folículos vitelogênicos ocorreram ao longo do ano em D. neivai (Fig. 2, $\chi^{2}=13,623 ; \mathrm{df}=11 ; \mathrm{p}>0,05$ ) e D. catesbyi (Fig $\left.3 ; \chi^{2}=13,352 ; \mathrm{df}=11 ; \mathrm{p}>0,05\right)$ e foram distribuídos de modo uniforme. Da mesma forma houve fêmeas maduras em diferentes fases do ciclo reprodutivo e as fêmeas prenhes ou fêmeas pós-postura apresentaram folículos em vários estágios de desenvolvimento, em ambas as espécies. As fêmeas prenhes ou com folículos ovulatórios apresentaram depósito de gordura no abdômen.

Dipsas neivai apresentou ovos nos ovidutos de maio a dezembro, exceto junho (Fig. 2). Foram registradas desovas em cativeiro em outubro $(n=2)$ e setembro e eclosões nos meses
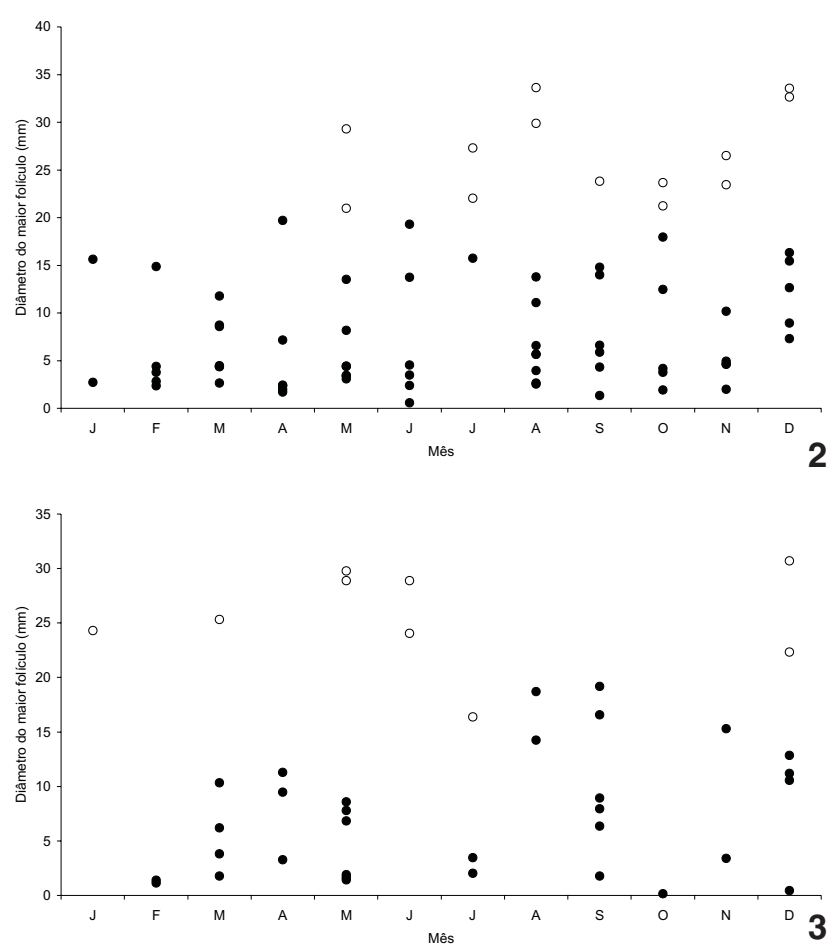

Figuras 2-3. Distribuição mensal do diâmetro do maior folículo ovariano (circulos preto) e comprimento do maior ovo (círculos branco) em (2) Dipsas neivai e (3) Dipsas catesbyi no sudeste da Bahia.

de janeiro, abril e dezembro. Os registros da coleção indicam que os espécimes com CRC $<240 \mathrm{~mm}$ (maior tamanho registrado no recém-nascido) foram coletados em janeiro, abril, junho, julho, agosto, setembro e outubro.

Dipsas catesbyi apresentou ovos nos ovidutos em janeiro, março, maio, junho, julho e dezembro (Fig. 3). As desovas em cativeiro ocorreram em março, agosto e outubro e as eclosões em fevereiro. Os registros da coleção indicam que espécimes com CRC < $202 \mathrm{~mm}$ (maior tamanho registrado no recém-nascido) foram coletados em fevereiro, março, maio, julho, agosto, outubro, novembro e dezembro.

\section{Tamanho da ninhada e do recém-nascido}

O tamanho da ninhada em $D$. neivai foi de um a oito ovos $(3,91 \pm 1,95 ; \mathrm{n}=22)$ e em $D$. catesbyi de um a seis de ovos $(3,63 \pm 1,66 ; n=13)$ e foi positivamente correlacionado com o CRC da fêmea em D. neivai (Fig. 4; $\mathrm{r}^{2}=0,6325 ; \mathrm{p}<0,05$ ), mas não em $D$. catesbyi (Fig. $\left.5 ; \mathrm{r}^{2}=0,51 ; \mathrm{p}>0,05\right)$. Em D. neivai o comprimento médio do ovo foi de $27,60 \pm 6,30 \mathrm{~mm}(19,50$ $41,50 \mathrm{~mm}, \mathrm{n}=90$ ovos de 24 ninhadas) e em $D$. catesbyi, 25,00 $\pm 3,02 \mathrm{~mm}(18,72-30,70 \mathrm{~mm}, \mathrm{n}=47$ ovos de 13 ninhadas $)$. $\mathrm{O}$ tamanho da ninhada não está correlacionado com o comprimento dos ovos de D. neivai (Fig. 6; $\mathrm{r}=0,032 ; \mathrm{p}>0,05$ ) ou $D$. catesbyi (Fig. 7; $\mathrm{r}=-0,05 ; \mathrm{p}>0,05$ ). 

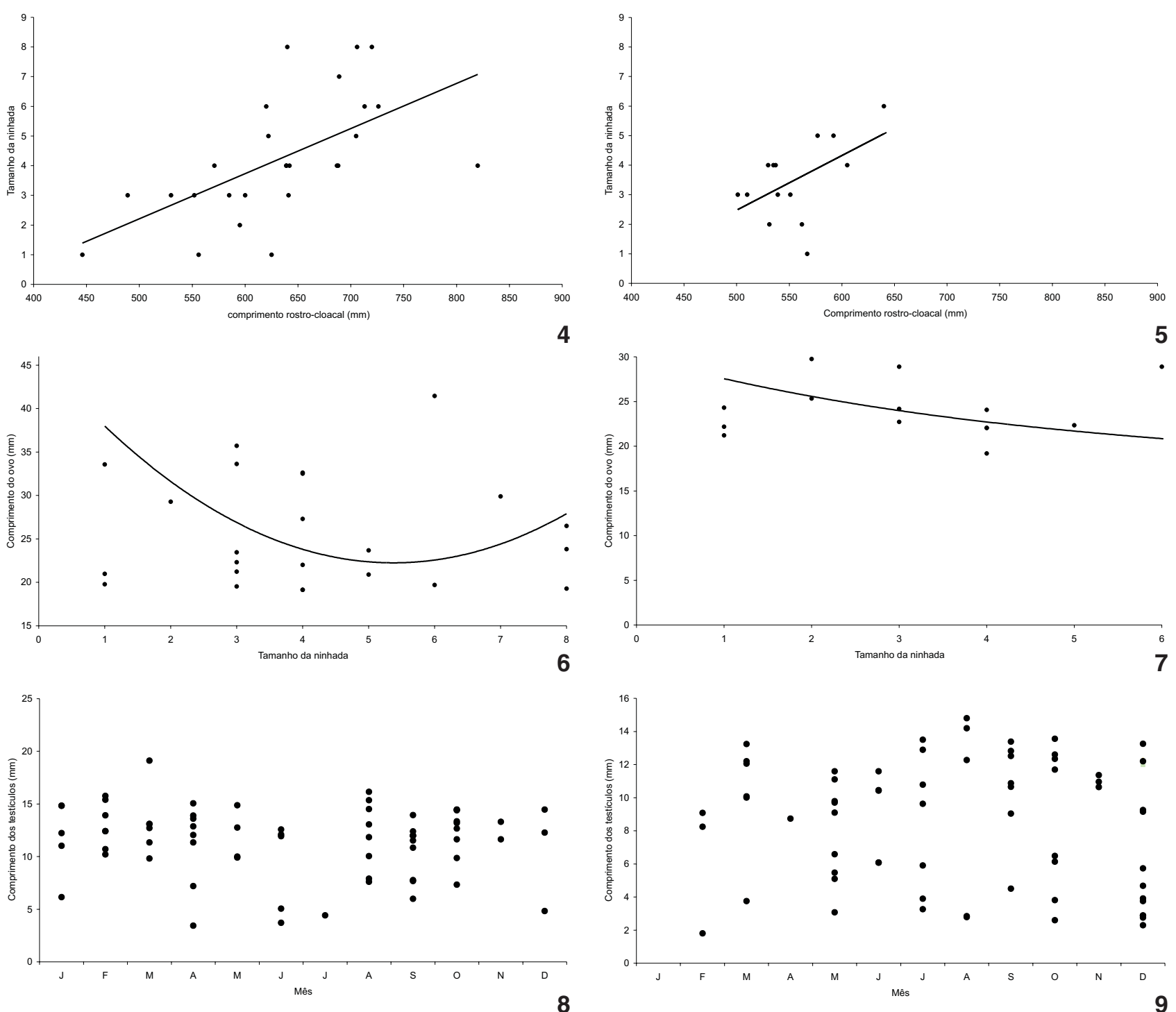

Figuras 4-9. (4-5) Relacionamento entre o comprimento rostro-cloacal e o tamanho da ninhada em (4) $D$. neivai $\left(r^{2}=0,63 ; p<0,05\right)$ e (5) D. catesbyi $\left(r^{2}=0,51 ; p>0,05\right) ;(6-7)$ relacionamento entre o tamanho da ninhada e o comprimento do ovo em (6) $D$. neivai $(r=$ $0,032 ; p>0,05)$ e $(7) D$. catesbyi $(r=-0,05 ; p>0,05) ;(8-9)$ comprimento dos testículos dos machos maduros de (8) $D$. neivai e (9) $D$. catesbyi ao longo do ano. $D$. neivai $(\mathrm{F}=0,669 ; \mathrm{df}=10 ; \mathrm{p}<0,05 ; \mathrm{n}=58)$ e $D$. catesbyi $(\mathrm{F}=0,487 ; \mathrm{df}=10 ; \mathrm{p}<0,05 ; \mathrm{n}=58)$.

Em cativeiro os ovos foram postos a intervalos de dois, três e até 19 dias. O período de incubação dos ovos de uma postura em cativeiro foi 90 dias. O recém-nascido em $D$. neivai mede $194,90 \mathrm{~mm} \pm 45,42(96-225 \mathrm{~mm} ; \mathrm{n}=13)$ e em $D$. catesbyi $188,07 \mathrm{~mm} \pm 10,52(170-202 \mathrm{~mm}, \mathrm{n}=24)$. Os recém-nascidos medem entre 16,43 e $29,27 \%$ do CRC materno em $D$. neivai e entre 31,56 e $49,42 \%$ do CRC materno em D. catesbyi.

\section{Machos}

Machos com testículos pequenos apresentaram ductos deferentes translúcidos e não enovelados em D. neivai ( $\mathrm{r}=0.546$; $\mathrm{p}>0,01 ; \mathrm{n}=112)$ e em $D$. catesbyi $(\mathrm{r}=0.696 ; \mathrm{p}>0,01 ; \mathrm{n}=95)$. Os comprimentos dos testículos dos indivíduos maduros de $D$. neivai $(\mathrm{F}=0,669 ; \mathrm{df}=10 ; \mathrm{p}>0,05 ; \mathrm{n}=58)$ e de D. catesbyi $(\mathrm{F}=$ 0,$487 ; \mathrm{df}=10 ; \mathrm{p}>0,05 ; \mathrm{n}=58$ ) não variaram ao longo do ano (Figs 8-9). O mesmo padrão foi observado para o diâmetro dos ductos deferentes de $D$. neivai $(\mathrm{F}=0,828 ; \mathrm{df}=10 ; \mathrm{p}>0,05 ; \mathrm{n}=$ $58)$ e de $D$. catesbyi $(\mathrm{F}=0,840 ; \mathrm{df}=10 ; \mathrm{p}>0,05 ; \mathrm{n}=58)$ (Figs 10 11), indicando ciclo contínuo em ambas as espécies.

A presença de machos e de fêmeas adultas de $D$. neivai $\left(\chi^{2}=13,925 ; \mathrm{df}=11 ; \mathrm{p}>0,05\right)$ e de $D$. catesbyi $\left(\chi^{2}=8,867 ; \mathrm{df}=\right.$ 

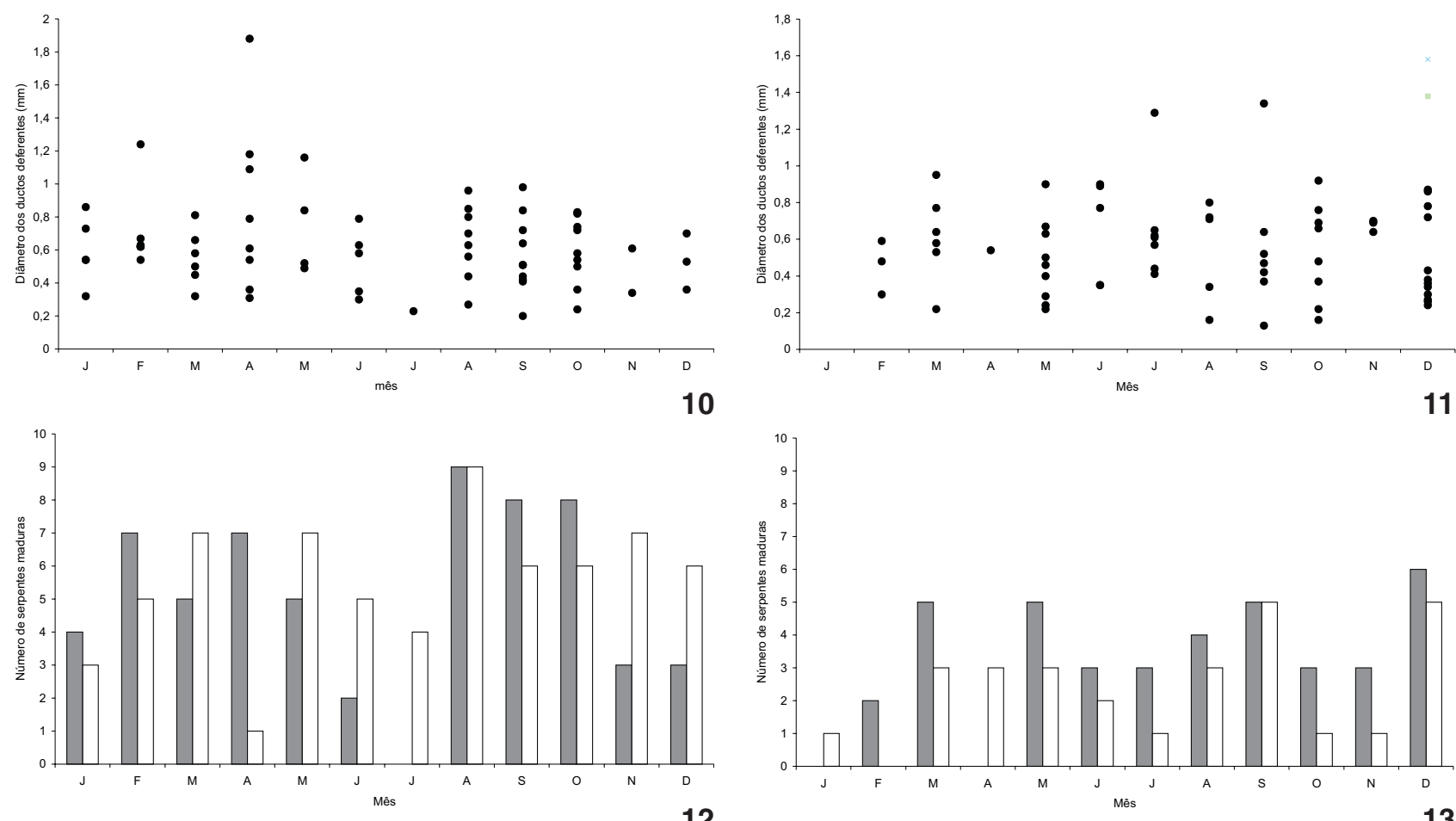

Figuras 10-13. (10-11) Diâmetro dos ductos deferentes em machos maduros de (10) D. neivai e (11) D. catesbyi ao longo do ano. $D$. neivai $(\mathrm{F}=0,828 ; \mathrm{df}=10 ; \mathrm{p}>0,05 ; \mathrm{n}=58)$ e $D$. catesbyi $(\mathrm{F}=0,840 ; \mathrm{df}=10 ; \mathrm{p}>0,05 ; \mathrm{n}=58) ;(12-13)$ distribuição mensal de machos (barras cinzas) e fêmeas adultos (barras brancas) de (11) D. neivai e (12) D. catesbyi em material colecionado no sudeste da Bahia.

11; $\mathrm{p}>0,05)$ foi registrada ao longo do ano com distribuição praticamente uniforme (Figs 12-13).

\section{Cópula}

Um casal de $D$. neivai em cópula foi observado em janeiro de 1992 , às 08:40 h da manhã sobre um cacaueiro, em Uruçuca, Bahia. O macho mediu $700 \mathrm{~mm}$ de CRC e a fêmea $550 \mathrm{~mm}$. O casal foi mantido em cativeiro durante três meses e sacrificado em 14 de abril de 1992. A fêmea apresentou folículos imaturos (maior folículo $=2,73 \mathrm{~mm}, \mathrm{n}=12$ ) e o macho apresentou testículos maduros $(\mathrm{CTE}=11,04 \mathrm{~mm})$ e ductos deferentes enovelados (diâmetro $=0,73 \mathrm{~mm}$ ).

Outra fêmea e três machos foram encontrados em fevereiro do mesmo ano, às 10:20h, enrodilhados sob as cascas de um tronco em decomposição, a 1,5 m do solo, no interior de um cacaual em Ilhéus-BA. Os machos mediram respectivamente 595,580 e $536 \mathrm{~mm}$ e a fêmea $580 \mathrm{~mm}$. Os machos apresentaram testículos maduros $(\mathrm{CTE}=10,72 ; 13,93$ e 10,20 mm e ductos deferentes enovelados (diâmetro $=0,62 ; 0,63$ e 0,54 mm). O exame histológico em um dos espécimes revelou espermatozóides no lúmen dos túbulos seminíferos (F. Q. Alves, dados não publicados). A fêmea apresentou folículos imaturos (maior folículo: 2,84 mm; $\mathrm{n}=38$ ) mas ovidutos espessos e com regiões convolutas.

\section{DISCUSSÃO}

Em D. neivai os machos amadurecem com menor tamanho que as fêmeas, como em outros colubrídeos ovíparos, a exemplo de Simophis rhinostoma (Schlegel, 1837) (Jordão \& BizerRa 1996); Erythrolamprus aesculapii (Linnaeus, 1758) (MARQUEs 1996) e Dipsas albifrons (HARTMANN et al. 2002). Esse padrão (e.g. PARKer \& Plummer 1987) é encontrado em 66\% das espécies de regiões temperadas. Para $D$. neivai, assim como em outras espécies em que a fecundação está correlacionada com o CRC (e.g. SHINE 1993), o maior tamanho confere vantagem seletiva para a fêmea. Isso se reflete no dimorfismo sexual, mais evidente nos adultos, com as fêmeas sendo maiores que os machos.

Machos de D. catesbyi amadurecem com maior CRC do que as fêmeas. Ou seja, contrariamente a $D$. neivai, fêmeas de D. catesbyi não retardam a reprodução para alcançar maior tamanho. Talvez isso explique a ausência de correlação entre o tamanho da ninhada e o CRC materno na espécie.

Em ambas as espécies as ninhadas são pequenas, um traço comum com outros Dipsadini (e.g. Duellman 1978, Zug et al. 1979, Manzani \& Cardoso 1997, Martins \& Oliveira 1998, HarTMANN et al. 2002). Nos representantes dessa tribo, o tamanho da ninhada tem sido relacionado aos hábitos semi-arborícolas (e.g. Martins \& Oliveira 1998) ou, ainda, ao bem estar físico da 
fêmea (Zug et al. 1979). Isso também pode se aplicar às espécies de Dipsas aqui estudadas. Entretanto, de acordo com SHINe (2003), o tamanho da fêmea determina a massa da ninhada somente se ela retarda a reprodução até obter energia suficiente para preencher sua cavidade com ovos ou embriões. Essa suposição se enquadra perfeitamente à situação de $D$. catesbyi.

O ciclo reprodutivo de $D$. neivai e $D$. catesbyi no sudeste da Bahia é contínuo. Isso coincide com o observado em $D$. catesbyi em Iquitos no Peru (Zug et al. 1979) e em D. neivai (Porto \& Fernandes 1996) na Mata Atlântica. Mesmo nos trópicos pode haver sazonalidade na reprodução de algumas espécies, devido a alguma variação nos níveis de recursos (SHINE 2003). Entretanto, a área estudada apresenta grande estabilidade climática (SÁ et al. 1982), o que também deve ocasionar disponibilidade contínua de presas no ambiente. Já Dipsadini ocorrendo em latitudes elevadas, a exemplo de D. albifrons (HARTMANN et al. 2002), pode haver sazonalidade no ciclo reprodutivo. Nestes casos a reprodução em geral ocorre na estação chuvosa, quando a disponibilidade de alimento e temperatura para incubação são mais adequadas (SHINE 2003), o que se reflete também na própria atividade das serpentes (SEIGel \& Ford 1987). Esse parece ser o caso, também, de populações mais meridionais de $D$. indica petersi (Marques \& SAZIMA 2004). D. neivai e D. catesbyi são as serpentes do gênero mais abundantes na região estudada e a estabilidade climática e a oferta de recursos podem ser responsáveis por permitir que espécies abundantes e com ciclo reprodutivo contínuo ocorram em sintopia.

A ocorrência de fêmeas prenhes ou em pós postura com folículos em diferentes estágios de desenvolvimento, sugere que $D$. neivai e $D$. catesbyi podem talvez produzir ninhadas múltiplas. Reservas de gordura, disponibilidade de alimento e a estrutura da população são os primeiros determinantes da freqüência reprodutiva em serpentes (SEIGEL \& FoRD 1987). Fêmeas prenhes das espécies aqui estudadas apresentaram presas não digeridas no estômago e depósitos de gordura abdominal. Isso demonstra que elas obtêm energia para a reprodução através da alimentação contínua (e.g. SHINE \& MADSEN 1997, BRown \& SHINE 2002) possuindo uma estrutura fisiológica adequada a produção de ninhadas múltiplas.

$\mathrm{O}$ acasalamento observado em $D$. neivai demonstrou que fêmeas apresentam a cópula dissociada da vitelogênese, embora os machos apresentem associação entre cópula e espermatogênese. Existem muitas espécies em que um ou ambos os sexos exibem dissociação entre a atividade gonadal e o acasalamento (Crews 1984). Há também evidências de que o ato de acasalamento estimula a vitelogênese (e.g. SEIGEL \& FORD 1987) e a dissociação entre cópula e vitelogênese pode estar relacionada ao estímulo desta nas fêmeas. Serpentes como Oxyrhopus guibei Hoge e Romano, 1977 (Pizzatto \& Marques 2002) estocam esperma e isso poderia ser uma explicação para a dissociação entre cópula e vitelogênese. Entretanto, ao contrário de $O$. guibei, machos e fêmeas sexualmente maduros de $D$. neivai e $D$. catesbyi foram encontrados em proporções seme- lhantes ao longo de todo o ano, tornando desnecessário o armazenamento de esperma.

\section{AGRADECIMENTOS}

Agradecemos a Otávio A. V. Marques e José V. Ortiz pela leitura crítica do manuscrito e ao segundo também pelo auxilio nas análises estatísticas. A Christine Strüssman e um revisor anônimo pelas valiosas sugestões ao texto. Ao Conselho Nacional de Desenvolvimento Científico e Tecnológico-CNPq pela bolsa de mestrado concedida a primeira autora. À Comissão Executiva do Plano da Lavoura Cacaueira (CEPLAC), Ilhéus-BA pelo empréstimo dos espécimes analisados. Este artigo é parte da dissertação de mestrado defendida no Curso de Pós-graduação e Zoologia da Universidade Estadual Paulista (UNESP), Botucatu, São Paulo.

\section{REFERÊNCIAS BIBLIOGRÁFICAS}

Amaral, A. Do. 1926. Nomes vulgares de Ophídios no Brasil. Boletim do Museu Nacional do Rio de Janeiro, Rio de Janeiro, 22 (2): 19-31.

Amaral, A. Do. 1978. Serpentes do Brasil. Iconografia Colorida. São Paulo, Edições Melhoramentos, Editora da Universidade de São Paulo, $2^{\text {a }}$ ed., 246p.

ARgôlo, A.J.S. 2004. As serpentes dos cacauais do sudeste da Bahia. Ilhéus, Bahia, Editus, 260p.

Brown, G.P. \& R. ShINe. 2002. Reproductive ecology of a tropical natricine snake, Tropidonophis mairii (Colubridae). Journal of Zoology, London, 258: 63-72.

Crews, D. 1984. Gamete production, Sex hormone secretion, and mating behavior uncoupled. Hormones and Behavior, Oxford, 18: 22-28.

Cunha, O.R. DA \& F.P. Nascimento. 1978. Ofídios da Amazônia. X. As cobras da região Leste do Pará. Publicações Avulsas do Museu Paraense Emílio Goeldi, Belém, 31: 1-218.

DuelLman, W.E. 1978. The biology of an equatorial herpetofauna in amazonian Ecuador. The University of Kansas Museum of Natural History, Miscellaneous Publications, Lawrence, 65: $1-352$.

Fiтch, H.S. 1981. Sexual size differences in reptiles. The University of Kansas Museum of Natural History, Miscellaneous Publications, Lawrence, 70: 1-72.

Hartmann, M.T.; M.L. del Grande; M.J. Gondim; M.C. Mendes \& O.A.V. MARQues. 2002. Reproduction and activity of the snaileating snake, Dipsas albifrons (Colubridae), in the southeastern Atlantic Forest in Brazil. Studies on Neotropical Fauna and Environment, Tübingen, 37: 111-114.

JoRdÃo, R. DOS S. \& A.L. BizerRa. 1996. Reprodução, dimorfismo sexual e atividade de Simophis rhinostoma (Serpentes, Colubridae). Revista Brasileira de Biologia, Rio de Janeiro, 56 (3): 507-512.

Martins, M. \& M.E. Oliveira. 1998: Natural history of snakes in forests of the Manaus region, central Amazonia, Brasil. 
Herpetological Natural History, La Sierra, 6: 78-150.

Manzani, P.R. \& A.J. CARDoso. 1997. Imantodes cenchoa (Chunkheaded snake). Reproduction. Herpetological Review, Saint Louis, 28: 154 .

MARQUeS, O.A.V. 1996a. Biologia reprodutiva da cobra-coral Erythrolamprus aesculapii, Linnaeus (Colubridae), no sudeste do Brasil. Revista Brasileira de Zoologia, Curitiba, 13: 747-753.

Marques, O.A.V. \& G. Puorto. 1998. Feeding, reproduction and growth in the crowned snake Tantilla melanocephala (Colubridae), from southeastern Brasil. Amphibia-Reptilia, Leiden, 19: 311-318.

Marques, O.A.V. \& I. Sazima. 2004. História natural dos répteis da Estação Ecológica Juréia-Itatins, p. 257-277. In: O.A.V. MARques \& W. Duleba (Eds). Estação Ecológica Juréia-Itatins. Ambiente físico, flora e fauna. Ribeirão Preto, Holos, 384p.

PArker, W.S. \& M.V. Plummer. 1987. Population Ecology, p. 253301. In: R.A. SeIgel; J.T. Collins \& S.S. NovaK (Eds). Snakes: ecology and evolutionary biology. New York, McGraw-Hill, $529 \mathrm{p}$.

Peters, J.A. \& B. Orejas-Miranda. 1970. Catalogue of The Neotropical Squamata: Part. I. Snakes. United States of Natural Museum Bulletin, Washington, 297: 1-347.

PizzatTo, L. \& O.A.V. Marques. 2002. Reproductive biology of the false coral snake Oxyrhopus guibei (Colubridae) from southeastern Brasil. Amphibia-Reptilia. Leiden, 23: 495-504.

Porto. M. \& R. Fernandes. 1996. Variation and Natural History of the snail-eating Snake Dipsas neivai (Colubridae Xenodontinae). Journal of Herpetology, New Haven, 30 (2): 269-271.

RoEdEr, M. 1975. Reconhecimento climatológico. Diagnóstico sócio-econômico da região cacaueira. Ilhéus, Convê- nio IICA/CEPLAC, vol. 4, 89p.

SÁ, D.F. de; H.A. de Almeida; L.F. da Silva \& A.C. LeÃo. 1987. Fatores edafo-climáticos seletivos ao zoneamento da cacauicultura. Revista Theobroma, Ilhéus, 12 (3): 169-187.

SEIGEL, R.A. \& N.B. FoRD. 1987. Reproductive ecology, p. 210252. In: R.A. SeIgel; J.T. Collins \& S.S NovaK (Eds). Snakes: ecology and evolutionary biology. New York, MacGrawHill, 529p.

SHINE, R. 1977a. Reproduction in australian elapid snakes II. Female reproductive cycles, Australian Journal of Zoology, Sydney, 25: 657-653.

SHINE, R. 1977b. Reproduction in australian elapid snakes. I. Testicular cycles and mating seasons. Australian Journal of Zoology, Sydney, 25: 647-653.

Shine, R. 1984. Reproductive biology and food habits of the Australian Elapid snakes of the genus Cryptophis. Journal of Herpetology, Athens, 18: 33-39

SHINE, R. 1993. Sexual dimorphism in snakes, p. 49-86. In: R.A. Seigel; J.T. Collins \& S.S NovaK (Eds). Snakes: ecology and behavior. New York, McGraw-Hill, 529p.

SHINE, R. 1994. Sexual size dimorphism in snakes revised. Copeia, Lawrence, 1994: 326-346.

SHINE, R. 2003. Reproductive strategies in snakes. The Royal Society Review, London, 270: 995-1004.

Shine, R. \& T. MAdsen. 1997. Prey abundance and predator reproduction: rats and pythons on a tropical Australian floodplain. Ecology, Washington, 78: 1078-1086.

Zug, G.R.; S.B.M. Hedges \& S. SunKel. 1979. Variation in reproductive parameters of tree neotropical snakes, Coniophanes fissidens, Dipsas catesbyi and Imantodes cenchoa. Smithsonian Contributions Zoology, Washington, 300:1-20.

Recebido em 30.IX.2004; aceito em 19.VII.2005. 